\section{Efeitos da estrutura etária nos gastos com internação no Sistema Único de Saúde: uma análise de decomposição para duas áreas metropolitanas brasileiras}

\author{
Population aging effects on inpatient care \\ expenditures: a disaggregated analysis \\ for two Brazilian metropolitan areas
}

\author{
1 Centro de Desenvolvimento \\ e Planejamento Regional, \\ Universidade Federal de \\ Minas Gerais, Belo Horizonte, \\ Brasil. \\ Correspondência \\ S. Wajnman \\ Departamento de \\ Demografia, Centro \\ de Desenvolvimento e \\ Planejamento Regional, \\ Universidade Federal de \\ Minas Gerais. \\ Av. Antônio Carlos 6627, \\ Belo Horizonte, $M G$ \\ 31370-901, Brasil. \\ wajnman@cedeplar.ufmg.br
}

\begin{abstract}
This paper examines the impact of changes in population age distribution on inpatient care expenditures in Brazil. The authors use data from two highly distinct metropolitan areas, $\mathrm{Cu}$ ritiba (Paraná State) and Belém (Paraná State), in order to determine to what extent current differences in expenditures are explained by differences in: (i) age distributions, (ii) age-specific expenditures (price effects), and (iii) utilization rates (rate effects). The study also looks at the consequences of future changes in age distribution by simulating each of the effects (age distribution, price, and rate) under the projected population age distribution for Brazil in 2050. The results show that only $13 \%$ of current differences in health expenditures in Curitiba and Belém are explained by differences in age distribution. Most of the difference is due to price effect (72\%), probably because of large socioeconomic and epidemiological discrepancies in these two metropolitan areas. However, simulations for 2050 suggest that most future changes in inpatient care will be explained by population aging effects.
\end{abstract}

Health Expenditure; Age Distribution; Aging; Hospitalization
Cláudia Koeppel Berenstein

Simone Wajnman 1

\section{Introdução}

O envelhecimento populacional suscita uma série de questões com respeito à composição dos gastos sociais, entre as quais a preocupação com o aumento dos gastos da saúde 1,2. Os efeitos desse processo vêm sendo seriamente discutidos nos países desenvolvidos, cuja transição demográfica já se encontra em estágios adiantados. Nota-se especial preocupação dos países que experimentaram o baby-boom, isto é, a descontinuidade populacional causada pelo aumento, sem precedentes, da fecundidade no pós-II Guerra Mundial. Esse fenômeno demográfico acentuou dramaticamente os contornos do envelhecimento da estrutura etária, ao exibir uma faixa etária desproporcionalmente mais numerosa - e também politicamente influente - que segue envelhecendo e trazendo à discussão a preocupação com o financiamento de suas demandas. À proporção que essa geração aproxima-se agora de idades mais elevadas, emergem as questões relacionadas às suas aposentadorias e às transformações no perfil de sua demanda por serviços de saúde.

Do ponto de vista da saúde, a transição demográfica está diretamente relacionada à transição epidemiológica, que se caracteriza, grosso modo, pela alteração do perfil de morbimortalidade antes marcado pela alta prevalência de doenças transmissíveis, para o predomínio das doenças crônico-degenerativas e causadas por fatores ex- 
ternos. Nesse processo, modifica-se paulatinamente o perfil de saúde da população: ao invés de processos agudos que se resolvem rapidamente por meio da cura ou do óbito, tornam-se predominantes as doenças crônicas e suas complicações, que muitas vezes significam décadas de utilização dos serviços de saúde - medicamentos, consultas médicas e internações hospitalares de longa duração, resultando, conseqüentemente, em maiores gastos ${ }^{3}$. São exemplos das enfermidades características deste novo perfil seqüelas do acidente vascular cerebral (AVC), fraturas após quedas e limitações provocadas pela insuficiência cardíaca e doença pulmonar obstrutiva crônica, além de amputações e cegueira provocadas pelo diabetes, e a dependência determinada pela demência de Alzheimer ${ }^{4}$. Além disso, verifica-se que quanto pior o status sócio-econômico maior tende a ser a procura por hospitais para utilização de serviços de alto custo ${ }^{5}$.

Na literatura internacional sobre os efeitos do envelhecimento populacional e da mudança do perfil epidemiológico sobre os gastos com saúde, apontam-se dois mecanismos distintos e complementares. Em primeiro lugar, como os gastos com tratamentos dispensados aos idosos tendem a ser mais caros, o aumento da proporção de idosos onera o sistema por um simples efeito de composição. Nessa linha, Cutler \& Meara 6 e Jacobzone \& Oxley 7 argumentam que o aumento dos gastos está altamente correlacionado com o uso intensivo de tecnologia em idades mais avançadas, acarretando a acentuação do chamado "efeito tecnológico". O maior uso de tecnologia no tratamento de doenças características dos idosos, como câncer e doenças cardiovasculares, implica um maior gasto com os grupos etários mais avançados, já que, de forma geral, quanto maior o emprego de tecnologia maiores os custos. Assim, conforme argumentam Pol \& Thomas 8, quando as doenças crônicas tornamse predominantes em uma população e sendo estas doenças diretamente relacionadas com a idade e o estilo de vida, a composição demográfica torna-se um aspecto chave para a análise da saúde de uma sociedade.

O segundo mecanismo de elevação dos gastos da saúde ocorre como conseqüência indireta do envelhecimento populacional, e é causado por aquilo que Buchner ${ }^{9}$ denomina de steeping (aumento da inclinação) da curva de gastos por idade. Esse mecanismo é explicado pelo crescimento mais rápido dos custos entre os grupos de idade mais velhos do que entre os grupos mais jovens. Analisando dados para a Alemanha, entre os anos de 1979 e 1996, Buchner ${ }^{9}$ verifica um forte aumento da inclinação da curva de gastos, $o$ que sugere grande impacto nas projeções futuras dos gastos com a saúde. Cutler \& Meara 6 analisam a evolução dos gastos de saúde nos Estados Unidos entre as décadas de 50 e 80 e identificam também uma forte elevação na inclinação à direita do perfil etário dos gastos. Ressaltam que, por outro lado, há uma concomitante elevação temporal dos gastos com os recém-nascidos, como conseqüência do emprego de tecnologias neonatais cada vez mais sofisticadas e onerosas.

Outros autores, no entanto, tendem a relativizar a noção de que a distribuição etária dos gastos com a saúde tenda a tornar-se crescentemente concentrada nos mais idosos em países cujos sistemas de saúde são mais avançados no emprego de tecnologia. Sheiner 10 verifica, por meio de informações para diversos países desenvolvidos, que os idosos mais velhos (the oldest old) recebem menos tecnologia em seus tratamentos do que os menos idosos, e sugere que uma população mais longeva pode implicar menores gastos com a saúde. Miller 11 corrobora essa noção e, analisando informações sobre o $\mathrm{Me}$ dicare americano em 1995, verifica que, quanto mais elevada a idade de morte menores os gastos despendidos: pessoas que morreram aos 75 anos, por exemplo, incorreram em um gasto médio de US\$ 13.500, enquanto uma morte aos 95 anos representou um gasto médio de US\$7.000. Esse declínio dos gastos com saúde com a maior longevidade dos idosos teria relação, segundo os autores citados, com o tipo de tratamento destinado aos pacientes, uma vez que os mais velhos tendem a ser tratados com menor agressividade do que os mais novos. Ademais, a probabilidade de um indivíduo mais velho morrer aumenta de uma forma geral, o que diminui, em média, o tempo de tratamento, assim como o número de dias de internação.

É preciso considerar ainda que medidas preventivas podem fazer com que os gastos com as doenças típicas dos idosos diminuam, caso seja possível evitar ou retardar o aparecimento de doenças que implicam gastos em recursos tecnológicos mais sofisticados e um maior tempo de permanência em hospitais. Assim, Spillman 12 argumenta que, se o envelhecimento populacional for acompanhado de uma grande melhora nas condições de saúde dos idosos, o impacto do envelhecimento nos gastos deverá ser moderado. Algumas evidências apontam que é justamente esse o caso da Inglaterra, onde, ao contrário do que ocorre em países como Japão, Canadá e Estados Unidos, tem-se verificado queda no gasto per capita da saúde dos idosos, enquanto os demais grupos etários experimentam aumento dos gastos 13 .

Nos países em desenvolvimento, o processo de envelhecimento populacional começou mais 
tarde, mas está ocorrendo em ritmo muito mais acelerado do que nos países desenvolvidos. No caso específico do Brasil, a população vem envelhecendo rapidamente há algumas décadas, desde que, na segunda metade dos anos sessenta, a queda das taxas de fecundidade criou as condições para o estreitamento progressivo da base da pirâmide populacional 14. Desse modo, a proporção de idosos na população, que não passava de $2,5 \%$ do total populacional na década de sessenta, está projetada para atingir os 15\% em aproximadamente vinte anos, de acordo com projeções do Instituto Brasileiro de Geografia e Estatística (IBGE). No plano da saúde, esse acelerado ritmo de envelhecimento cria uma situação bem identificada por Saad 15, em que se superpõem as demandas típicas dos países mais envelhecidos e os desafios do atendimento à saúde materno-infantil e às doenças transmissíveis que ainda persistem no país, sobretudo nas regiões mais pobres. Por outro lado, o Brasil é um país de grande heterogeneidade regional, com áreas de pobreza absoluta e subdesenvolvimento, coexistindo com regiões bastante industrializadas nas quais o nível de renda e de consumo assemelha-se ao dos países mais desenvolvidos. A oferta de serviços de saúde acompanha essa disparidade regional, que se reflete tanto no acesso da população aos serviços quanto no estado de saúde das pessoas que vão procurar atendimento. Conseqüentemente, o perfil de morbidade, assim como a estrutura de gastos com a saúde, varia, não só com o grau de envelhecimento populacional, mas também de acordo com as condições sócio-econômicas e de oferta de serviços de cada região 16 .

O objetivo deste artigo é estimar o papel da composição etária da população sobre os gastos com internação hospitalar no Brasil, contemplando o efeito desta diversidade regional por meio da análise de dados do sistema de informação hospitalar de duas cidades (Curitiba, Paraná e Belém, Pará), em estágios distintos do processo de envelhecimento populacional e desenvolvimento sócio-econômico, em 2004.

\section{Metodologia}

A diferença nos gastos totais com internação hospitalar entre Curitiba e Belém será decomposta em: (i) efeito de diferenças na composição etária; (ii) efeito preço (diferenças nos gastos médios por idade); e (iii) efeito taxa (diferenças na taxas de utilização por idade). Além disso, assumindo que os gastos médios e as taxas de utilização por idade serão constantes no futuro, o artigo examina o efeito do envelhecimento populacional sobre os gastos de internação com base nas mudanças projetadas para a composição etária do Brasil em 2050.

Duas capitais brasileiras com características diametralmente opostas foram escolhidas para as comparações feitas neste artigo. A primeira, Curitiba, localizada na Região Sul, exibe indicadores sociais e econômicos melhores do que os do restante do país, assim como uma melhor oferta de serviços de saúde. A segunda capital, Belém, localiza-se numa das regiões mais atrasadas do Brasil, onde tanto a oferta quanto o acesso aos serviços de saúde são bastante precários. Apenas para citar alguns dados, em 2000, Curitiba apresentou um Índice de Desenvolvimento Humano (IDH) de 0,856 e esperança de vida ao nascer de 71,6 anos, enquanto em Belém os valores foram de 0,806 e 70,5, respectivamente (Atlas do desenvolvimento humano no Brasil. http://www. pnud.org.br/atlas, acessado em 15/Jul/2007). O diferencial na oferta de saúde é ainda mais evidente: enquanto Curitiba, em 1999, apresentava 2,12 médicos disponíveis no SUS por mil habitantes, Belém apresentava apenas 1,31 17.

Para a análise dos gastos de saúde foram utilizados os dados das Autorizações de Internação Hospitalar (AIH), no ano de 2004, provenientes do Sistema de Informações Hospitalares (SIH), disponibilizados pelo Ministério da Saúde 18. A cada internação ocorrida pelo SUS é preenchida uma AIH, que consiste em um formulário com informações sobre o hospital que efetua o atendimento, dados de identificação do paciente, causa da internação, valor pago pelo procedimento, entre outros. O estabelecimento hospitalar envia as informações das AIH para os gestores municipais ou estaduais, que as utilizam para o acompanhamento dos procedimentos realizados durante a internação do paciente e para o pagamento aos prestadores dos serviços. Essas informações são processadas pelo Departamento de Informática do SUS (DATASUS), gerando os créditos referentes aos serviços prestados e formando a base de dados do Sistema de Informações Hospitalares do SUS (SIH/SUS). O SIH/SUS é considerado uma valiosa fonte de informações, tanto para pesquisas epidemiológicas (estudo de mortalidade/morbidade hospitalar, avaliação de serviços) quanto para fiscalização e auditoria do Ministério da Saúde.

No que tange à população observada nas duas capitais, que corresponde ao denominador das taxas utilizadas neste artigo, foram utilizadas as estimativas do sistema de informações do DATASUS. Vale ressaltar que a população observada não é exatamente a usuária do SUS, e com isso inclui-se também a população que utiliza o sistema suplementar, problema que certamente 
será maior no caso de Curitiba, já que maior parte da população desta cidade tem acesso à saúde particular. Com isso, as taxas que utilizam como denominador a população observada não são exatamente uma medida de utilização do SUS e devem ser analisadas como uma razão entre internações no SUS e população total.

Como as AIH restringem-se aos procedimentos realizados apenas em internações, todos os gastos ambulatoriais e com medicamentos não são incluídos nesta análise. É importante ressalvar também que as AIH informam apenas sobre as internações financiadas pelo SUS, ou seja, pagas pelo serviço público de saúde, deixando de incluir qualquer internação ocorrida no âmbito particular ou financiada por planos de saúde. No entanto, as internações financiadas pelo sistema público parecem representativas do padrão de gastos e de causas de morbidade da população, uma vez que $72 \%$ da população brasileira utilizam os serviços de saúde oferecidos pelo SUS como única fonte de atenção à saúde 19.

Para o ano de 2004, as AIH registram, para o sexo masculino, 48.112 internações em Belém e 69.339 em Curitiba. Entretanto, as capitais tendem a atrair residentes de localidades menores e mais deficientes em serviços públicos de saúde, que se deslocam em busca de atendimento. Dessa forma, e devido à utilização da população do município no denominador com as ressalvas já citadas, foram utilizados somente os dados relativos aos internados residentes no próprio município. Com isso, o universo de análise reduziu-se para 31.740 internações em Belém e 42.907 em Curitiba.

\section{Análise dos dados}

É possível decompor o gasto total das internações em três parcelas: preço, que é determinado pelo gasto médio das internações por grupo etário; $t a$ $x a$, relativa à taxa de utilização por grupo etário; e composição, que reflete a distribuição da população por faixa etária, conforme a equação 1:

$$
G T=\sum_{i} G M_{i} \times T U_{i} \times P_{i}
$$

Em que:

$G T$ : gasto total da internação;

$G M_{i}$ : gasto médio da internação para cada grupo etário i;

$T U_{i}$ : taxa de utilização de internação para cada grupo etário i;

$P_{i}$ : população residente para cada grupo etário i.

Dessa forma, diferenças de gastos totais entre localidades distintas, assim como mudanças no tempo, podem ser decompostas em efeito preço, efeito taxa e efeito composição. O efeito preço decorre de diferenças nos gastos médios por grupo etário, que refletem os procedimentos adotados e seus preços, dependendo fundamentalmente do perfil da oferta de serviços nas regiões analisadas. O efeito taxa corresponde ao grau de utilização dos serviços hospitalares e responde a variações no tipo de atendimento dado aos pacientes, dependendo do comportamento da demanda pelos serviços, influenciada tanto pelo perfil de morbidade da população quanto pela própria oferta, que condiciona a procura pelos serviços disponíveis à população. A taxa de utilização sofreria uma queda, por exemplo, se grande parte dos atendimentos antes resolvida apenas com a internação do paciente passasse a ser atendida pelo serviço ambulatorial. O efeito composição decorre de variações na estrutura etária da população, uma vez que estão sendo consideradas populações padronizadas pelo tamanho. No caso, a população de Belém foi padronizada segundo o tamanho da de Curitiba. Assim, o efeito composição reflete diferenças na estrutura etária, as quais dependem dos comportamentos da fecundidade e das taxas de mortalidade e migrações por idade. Somando-se os três efeitos obtém-se a diferença de gastos totais entre as duas capitais.

Portanto, o método aqui empregado consiste, em primeiro lugar, na análise dos componentes do gasto total por grupo etário, ou seja, gasto médio, taxa de utilização e estrutura etária. Em segundo lugar, faz-se a decomposição da diferença dos gastos totais de internação nos efeitos preço, taxa e composição, identificando-se quanto da diferença entre os gastos de internação de Belém e Curitiba é explicada por cada um dos efeitos. Finalmente, para isolar o potencial efeito composição de uma estrutura etária mais envelhecida, refaz-se o mesmo exercício de decomposição assumindo-se, para Curitiba, a estrutura etária que se projeta, de acordo com o IBGE (Projeções populacionais - revisão de 2004. http://www.ibge. gov.br, acessado em 18/Out/2004) para o Brasil em 2050. Dessa forma, procura-se medir a amplitude do efeito puro da mudança da estrutura etária que deverá ocorrer no Brasil nas próximas décadas.

\section{Análise dos componentes do gasto total por grupo etário}

Como o gasto total é determinado pela combinação entre gastos médios por idade, taxas de utilização e estrutura etária da população, procede-se a análise, por grupo etário, dos seguintes indicadores: 
a) Participação do grupo etário no gasto total:

Em que: ${ }_{n} \% G T_{x}=\frac{\sum_{n} G I_{x}}{\sum G I}$

${ }_{n} \% G T$ é o percentual do gasto total no grupo etário $\mathrm{x}$ a x+n;

$\sum_{n} G I_{x}$ é o somatório dos gastos de internação no grupo etário $\mathrm{x}$ a $\mathrm{x}+\mathrm{n}$;

$\sum$ GI é o somatório dos gastos de internação em todos os grupos etários.

Os demais indicadores correspondem às componentes do gasto total por idade, sendo eles: b) Gasto médio por grupo etário, dado por:

$$
{ }_{n} G M_{x}=\frac{{ }_{n} G I_{x}}{{ }_{n} N_{x}}
$$

Em que:

${ }_{n} G M_{x}$ é o gasto médio no grupo etário $\mathrm{x}$ a $\mathrm{x}+\mathrm{n}$;

${ }_{n} G I_{x}$ é o gasto de internação no grupo etário $\mathrm{x}$ a $\mathrm{X}+\mathrm{n}$;

${ }_{n} N_{x}$ é o número de internações no grupo etário $\mathrm{x} \mathrm{a} \mathrm{x+n.}$

A análise dos perfis etários de gastos médios permite identificar quais são os grupos cujos procedimentos têm gastos mais elevados. O efeito preço, analisado posteriormente, varia de acordo com o gasto médio.

c) Taxa de utilização por grupo etário:

A taxa de utilização mostra a freqüência de internação em cada grupo etário, indicando quais foram os grupos que mais utilizaram os serviços de internações no período analisado. O efeito ta$x a$ que será analisado posteriormente é determinado pelas variações da taxa de utilização.

$$
{ }_{n} T U_{x}=\frac{{ }_{n} N_{x}}{{ }_{n} P_{x}}
$$

${ }_{n} T U_{x}$ é a taxa de utilização no grupo etário x a $\mathrm{x}+\mathrm{n}$;

${ }_{n} N_{x}$ é o número de internações no grupo etário $\mathrm{x}$ a $\mathrm{x}+\mathrm{n}$;

${ }_{n} P_{x}$ é a população total no grupo etário $\mathrm{x}$ a $\mathrm{x}+\mathrm{n}$.

d) Estrutura etária da população, que determina o efeito composição, é dada por:

$$
{ }_{n} E E_{x}=\frac{{ }_{n} P_{x}}{P}
$$

${ }_{n} E E_{x}$ é a proporção da população no grupo etário $\mathrm{x} \mathrm{a} \mathrm{x+n;}$

${ }_{n} P_{x}$ é a população observada no grupo etário $\mathrm{x}$ $\mathrm{ax}+\mathrm{n}$;

$P$ é a população total observada.

\section{Decomposição do gasto total}

A decomposição do gasto total visa a identificar a participação relativa de cada uma das componentes acima descritas, sendo que tal decom- posição pode ser utilizada para avaliar os efeitos das componentes nas variações dos gastos tanto temporais quanto regionais. Neste trabalho, optou-se por tomar a comparação entre duas capitais com diferentes níveis de desenvolvimento sócio-econômico, no ano de 2004, como uma proxy para o efeito de mudanças temporais.

A decomposição do gasto total é feita utilizando-se método semelhante ao descrito por Preston et al. 20 para explicar a proporção das diferenças nas taxas brutas de mortalidade atribuída ao efeito composição e ao efeito taxa. Desse modo, define-se a diferença observada entre os gastos totais das duas capitais (Belém como população l e Curitiba como população 2) da seguinte maneira:

$\Delta=G T^{2}-G T^{1}=\sum_{x=0}^{80+}{ }_{n} G M_{x}^{2} \times{ }_{n} T U_{x}^{2} \times{ }_{n} P_{x}^{2}-\sum_{x=0}^{80+}{ }_{n} G M_{x}^{1} \times{ }_{n} T U_{x}^{1} \times{ }_{n} P_{x}^{1}$

Rearranjando os termos (para desenvolvimento da equação ver Figura 1) obtém-se as 3 componentes, as quais foram denominadas efe $i$ to preço, taxa e composição. Ou seja:
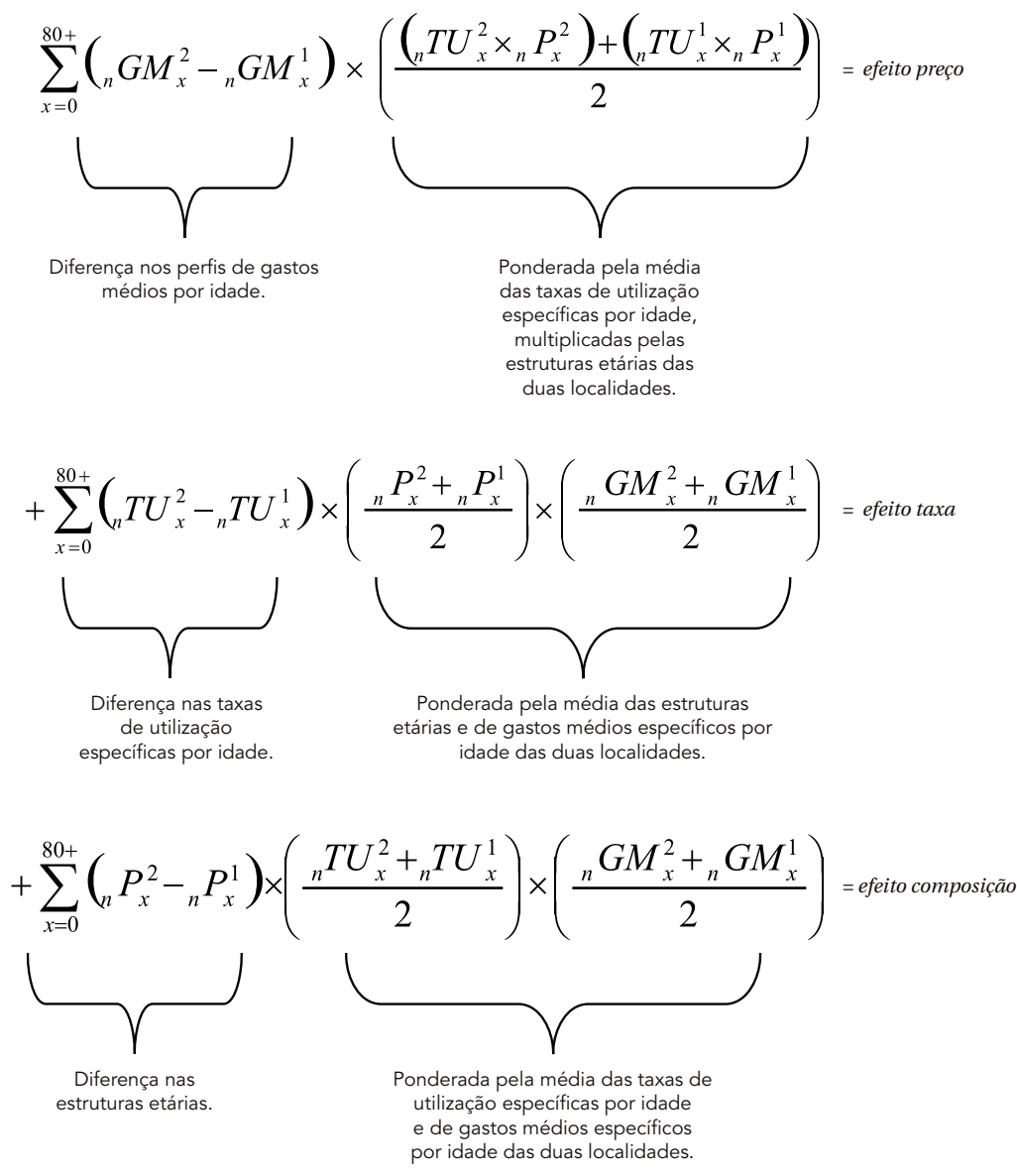

Assim, a diferença entre os gastos totais observados nas duas cidades $(\Delta)$ pode ser decomposta em: (i) contribuição da diferença de gasto 


$$
\begin{gathered}
\Delta=G T^{2}-G T^{1}=\sum_{x=0}^{80+}{ }_{n} G M_{x}^{2} \times{ }_{n} T U_{x}^{2} \times{ }_{n} P_{x}^{2}-\sum_{x=0}^{80+}{ }_{n} G M_{x}^{1} \times{ }_{n} T U_{x}^{1} \times{ }_{n} P_{x}^{1} \\
\text { Fazendo-se } \quad{ }_{n} T U_{x}^{2} \times{ }_{n} P_{x}^{2}={ }_{n} X_{x}^{2} \text { e }{ }_{n} T U_{x}^{1} \times{ }_{n} P_{x}^{1}={ }_{n} X_{x}^{1} \\
\Delta=G T^{2}-G T^{1}=\sum_{x=0}^{80+}{ }_{n} G M_{x}^{2} \times{ }_{n} X_{x}^{2}-\sum_{x=0}^{80+}{ }_{n} G M_{x}^{1} \times{ }_{n} X_{x}^{1}
\end{gathered}
$$

Dividindo-se estes dois termos em duas partes iguais e adicionando-se outros termos, mantendo a diferença constante, temos:

$$
\begin{gathered}
\Delta=\frac{\sum_{x=0}^{80+}{ }_{n} G M_{x}^{2} \times_{n} X_{x}^{2}}{2}+\frac{\sum_{x=0}^{80+}{ }_{n} G M_{x}^{2} \times{ }_{n} X_{x}^{2}}{2}-\frac{\sum_{x=0}^{80+}{ }_{n} G M_{x}^{1} \times{ }_{n} X_{x}^{1}}{2}-\frac{\sum_{x=0}^{80+}{ }_{n} G M_{x}^{1} \times{ }_{n} X_{x}^{1}}{2} \\
+\frac{\sum_{x=0}^{80+}{ }_{n} G M_{x}^{2} \times{ }_{n} X_{x}^{1}}{2}-\frac{\sum_{x=0}^{80+}{ }_{n} G M_{x}^{2} \times X_{n}^{1}}{2}+\frac{\sum_{x=0}^{80+}{ }_{n} G M_{x}^{1} \times{ }_{n} X_{x}^{2}}{2}-\frac{\sum_{x=0}^{80+}{ }_{n} G M_{x}^{1} \times{ }_{n} X_{x}^{2}}{2}
\end{gathered}
$$

Combinando-se agora os oito termos de $\Delta$ em quatro termos e depois em dois, tem-se:

$$
\begin{array}{r}
\Delta=\sum_{x=0}^{80+}{ }_{n} G M_{x}^{2} \times\left(\frac{{ }_{n} X_{x}^{2}+{ }_{n} X_{x}^{1}}{2}\right)-\sum_{x=0}^{80+}{ }_{n} G M_{x}^{1} \times\left(\frac{{ }_{n} X_{x}^{2}+{ }_{n} X_{x}^{1}}{2}\right) \\
+\sum_{x=0}^{80+}{ }_{n} X_{x}^{2} \times\left(\frac{{ }_{n} G M_{x}^{2}+{ }_{n} G M_{x}^{1}}{2}\right)-\sum_{x=0}^{80+}{ }_{n} X_{x}^{1} \times\left(\frac{{ }_{n} G M_{x}^{2}+{ }_{n} G M_{x}^{1}}{2}\right) \\
\Delta=\sum_{x=0}^{80+}\left(G M_{x}^{2}-{ }_{n} G M_{x}^{1}\right) \times\left(\frac{{ }_{n} X_{x}^{2}+{ }_{n}^{1}}{2}\right)+\sum_{x=0}^{80+}\left({ }_{n} X_{x}^{2}-{ }_{n} X_{x}^{1}\right) \times\left(\frac{{ }_{n} G M_{x}^{2}+{ }_{n} G M_{x}^{1}}{2}\right) \\
\text { Mas } \sum_{x=0}^{80+}\left({ }_{n} X_{x}^{2}-{ }_{n} X_{x}^{1}\right)=\sum_{x=0}^{80+} T{ }_{n} T U_{x}^{2} \times{ }_{n} P_{x}^{2}-\sum_{x=0}^{80+}{ }_{n} T U_{x}^{1} \times{ }_{n}^{1}
\end{array}
$$$$
\text { Repetindo-se o mesmo procedimento anterior, tem-se que: }
$$

$$
\begin{aligned}
& \sum_{x=0}^{80+}\left(X_{n}^{2} X_{x}-{ }_{n} X_{x}^{1}\right)=\frac{\sum_{x=0}^{80+}{ }_{n} T U_{x}^{2} \times_{n} P_{x}^{2}}{2}+\frac{\sum_{x=0}^{80+} T U_{x}^{2} \times_{n} P_{x}^{2}}{2}-\frac{\sum_{x=0}^{80+} T U_{x}^{1} \times{ }_{n} P_{x}^{1}}{2}-\frac{\sum_{x=0}^{80+}{ }_{n} T U_{x}^{1} \times{ }_{n} P_{x}^{1}}{2} \\
& +\frac{\sum_{x=0}{ }_{n} T U_{x}^{2} \times{ }_{n} P_{x}^{1}}{2}-\frac{\sum_{x=0}^{80+} T U_{x}^{2} \times{ }_{n} P_{x}^{1}}{2}+\frac{\sum_{x=0}^{80+} T U_{n}^{1} \times{ }_{n} P_{x}^{2}}{2}-\frac{\sum_{x=0}^{80+} T U_{x}^{1} \times{ }_{n} P_{x}^{2}}{2}
\end{aligned}
$$


Combinando-se novamente os oito termos da equação em quatro termos e depois em dois:

$$
\begin{gathered}
\sum_{x=0}^{80+}\left({ }_{n} X_{x}^{2}-{ }_{n} X_{x}^{1}\right)=\sum_{x=0}^{80+} T U_{x}^{2} \times\left(\frac{{ }_{n} P_{x}^{2}+{ }_{n} P_{x}^{1}}{2}\right)-\sum_{x=0}^{80+}{ }_{n} T U_{x}^{1} \times\left(\frac{{ }_{n} P_{x}^{2}+{ }_{n} P_{x}^{1}}{2}\right) \\
+\sum_{x=0}^{80+}{ }_{n} P_{x}^{2} \times\left(\frac{{ }_{n} T U_{x}^{2}+{ }_{n} T U_{x}^{1}}{2}\right)-\sum_{x=0}^{80+}{ }_{n} P_{x}^{1} \times\left(\frac{{ }_{n} T U_{x}^{2}+{ }_{n} T U_{x}^{1}}{2}\right) \\
\sum_{x=0}^{80+}\left({ }_{n} X_{x}^{2}-{ }_{n} X_{x}^{1}\right)=\sum_{x=0}^{80+}\left({ }_{n} T U_{x}^{2}-{ }_{n} T U_{x}^{1}\right) \times\left(\frac{{ }_{n} P_{x}^{2}+{ }_{n} P_{x}^{1}}{2}\right)+\sum_{x=0}^{80+}\left({ }_{n} P_{x}^{2}-{ }_{n} P_{x}^{1}\right) \times\left(\frac{{ }_{n} T U_{x}^{2}+{ }_{n} T U_{x}^{1}}{2}\right) \\
\text { Substituindo, na equação (5), os termos }{ }_{n} X_{x}^{2} \text { e }{ }_{n} X_{x}^{1} \text { e o termo }\left({ }_{n} X_{n}^{2}-{ }_{n} X_{x}^{1}\right) \text { pelo que foi obtido na equação (8), tem-se que: } \\
\Delta=\sum_{x=0}^{80+}\left({ }_{n} G M_{x}^{2}-{ }_{n} G M_{x}^{1}\right) \times\left(\frac{\left({ }_{n} T U_{x}^{2} \times{ }_{n} P_{x}^{2}\right)+\left({ }_{n} T U_{x}^{1} \times{ }_{n} P_{x}^{1}\right)}{2}\right)+ \\
{\left[\sum_{x=0}^{80+}\left({ }_{n} T U_{x}^{2}-{ }_{n} T U_{x}^{1}\right) \times\left(\frac{{ }_{n} P_{x}^{2}+{ }_{n} P_{x}^{1}}{2}\right)+\sum_{x=0}^{80+}\left({ }_{n} P_{x}^{2}-{ }_{n} P_{x}^{1}\right) \times\left(\frac{{ }_{n} T U_{x}^{2}+{ }_{n} T U_{x}^{1}}{2}\right)\right] \times\left(\frac{{ }_{n} C M_{x}^{2}+{ }_{n} C M_{x}^{1}}{2}\right)}
\end{gathered}
$$

$$
\begin{aligned}
& \sum_{x=0}^{80+}\left({ }_{n} G M_{x}^{2}-{ }_{n} G M_{x}^{1}\right) \times\left(\frac{\left({ }_{n} T U_{x}^{2} \times{ }_{n} P_{x}^{2}\right)+\left({ }_{n} T U_{x}^{1} \times{ }_{n} P_{x}^{1}\right)}{2}\right)=\text { efeito prę̧ } \\
& +\sum_{x=0}^{80+}\left({ }_{n} T U_{x}^{2}-{ }_{n} T U_{x}^{1}\right) \times\left(\frac{{ }_{n} P_{x}^{2}+{ }_{n} P_{x}^{1}}{2}\right) \times\left(\frac{{ }_{n} G M_{x}^{2}+{ }_{n} G M_{x}^{1}}{2}\right)=\text { efeito taxa } \\
& +\sum_{x=0}^{80+}\left({ }_{n} P_{x}^{2}-{ }_{n} P_{x}^{1}\right) \times\left(\frac{{ }_{n} T U_{x}^{2}+{ }_{n} T U_{x}^{1}}{2}\right) \times\left(\frac{{ }_{n} G M_{x}^{2}+{ }_{n} G M_{x}^{1}}{2}\right)=\text { efeito composiçäo }
\end{aligned}
$$


médio na diferença de gastos totais, ou seja, efeito preço; (ii) contribuição da diferença de taxa de utilização na diferença de gastos totais, ou seja, efeito taxa; (iii) contribuição da diferença de estrutura etária na diferença de gastos totais, ou seja, efeito composição.

Simulação do gasto total a partir da estrutura etária do Brasil em 2050

Para mensurar o potencial efeito composição decorrente do envelhecimento populacional na diferença de gastos totais, supôs-se que a estrutura etária de Curitiba fosse equivalente à do Brasil em 2050, e novamente fez-se a decomposição dos gastos como mencionado na seção anterior. Por meio desse exercício, mensura-se o potencial efeito puro da estrutura etária que o Brasil terá em 2050, quando sua transição demográfica já estiver completada.

\section{Resultados}

O Brasil apresenta ainda uma população relativamente jovem, desta forma espera-se que os gastos com grupos etários mais jovens sejam bastante onerosos para o sistema. Contudo, com o envelhecimento populacional, a parcela de gastos relativos aos grupos etários mais avançados vem aumentando de forma bastante acelerada. A Figura 2 mostra que a estrutura etária masculina de Curitiba é um pouco mais envelhecida que a de Belém. Porém, ambas ainda são bem jovens se comparadas à projetada para o Brasil em 2050. Quanto à taxa de utilização (Figura 3), é evidente que, entre todos os grupos etários, os que mais utilizam os serviços de internação são os menores de um ano e os idosos. Os menores de um ano representam 2,05\% da população de Belém e $1,68 \%$ da de Curitiba, mas a proporção dos gastos totais atribuída a este grupo etário é bem mais elevada, sendo de aproximadamente $13 \%$ para Belém e 9\% para Curitiba. Fato semelhante ocorre com os idosos. Em Belém eles correspondem a $5,84 \%$ da população, mas representam $25 \%$ do gasto total. Em Curitiba quase $27 \%$ do gasto total são relativos às pessoas com 60 anos e mais, embora estas representem, ainda, apenas 7,18\% da população total (Figura 4). Na descrição do perfil de gastos de internação, no gráfico 3, é possível perceber que Curitiba apresenta valores relativamente mais altos nas idades mais velhas em relação a Belém. Esse padrão pode ser explicado observando-se os demais gráficos. Os gastos médios (Figura 5) são mais elevados em Curitiba, provavelmente como resultado de um sistema de saúde tecnologicamente mais equipado. De fato, conforme sugere a literatura, as áreas mais desenvolvidas tendem a apresentar gastos mais elevados, especialmente para os menores de um ano e nas idades mais avançadas, devido a uma oferta mais ampla de serviços e de uma melhor infra-estrutura hospitalar.

Quando se decompõe o gasto total das internações em efeito preço, efeito taxa e efeito composição (Tabela 1) percebe-se que, para o ano de 2004 a diferença de gastos médios entre as duas capitais (efeito preço) é a principal responsável pela diferença no gasto total, explicando $72 \%$ da mesma. Pode-se dizer que, caso a estrutura de gastos médios por grupo etário fosse a mesma, a diferença nos gastos totais seria de apenas um terço do valor observado. A proporção atribuída ao efeito taxa é bem inferior, equivalendo a $15 \%$, e o efeito composição exerce uma pressão um pouco menor, $12,7 \%$, uma vez que a estrutura etária das capitais é bastante semelhante. Assim, conclui-se que a principal diferença nos gastos totais - padronizados pelo tamanho populacional - decorre da diferença entre as estruturas de oferta hospitalar de uma e outra capital, com a mais desenvolvida apresentando gastos médios muito mais elevados nos grupos extremos.

Para estimar o efeito potencial do envelhecimento populacional nos gastos das internações, simulou-se o gasto total de Curitiba adotandose a estrutura etária projetada para o Brasil em 2050, quando se espera que a transição demográfica esteja completada. O resultado obtido é que a proporção da diferença explicada pelo efeito composição, isto é, da diferença no gasto total explicado pelas mudanças na estrutura etária entre 2004 e 2050, aumenta para 54,9\%, enquanto os demais efeitos sofrem redução na parcela explicada, como mostra a Tabela 1 . O efeito preço, apesar de ainda exercer um grande papel, cai para quase metade do obtido no exercício anterior. É preciso notar, no entanto, que a simulação aqui realizada leva em conta apenas o efeito puro da estrutura etária. Evidentemente, não há nenhuma razão para se acreditar que o envelhecimento populacional não venha acompanhado de forte elevação nos gastos médios relativos aos mais idosos, sobretudo se mantido o atual modelo de saúde baseado em tratamentos curativos ao invés de preventivos.

\section{Discussão}

Este trabalho demonstra que os grupos etários que mais utilizam os serviços de internação no SUS são os menores de um ano de idade e os idosos. De fato, a curva de padrão de uso de serviços de saúde em forma de J já foi encontrada tanto em estudos nacionais quanto internacionais 17,21 . Para Curitiba, constatou-se que os gastos médios 
Estrutura etária de homens, em Belém (Pará) e Curitiba (Paraná), em 2004 e no Brasil em 2050.

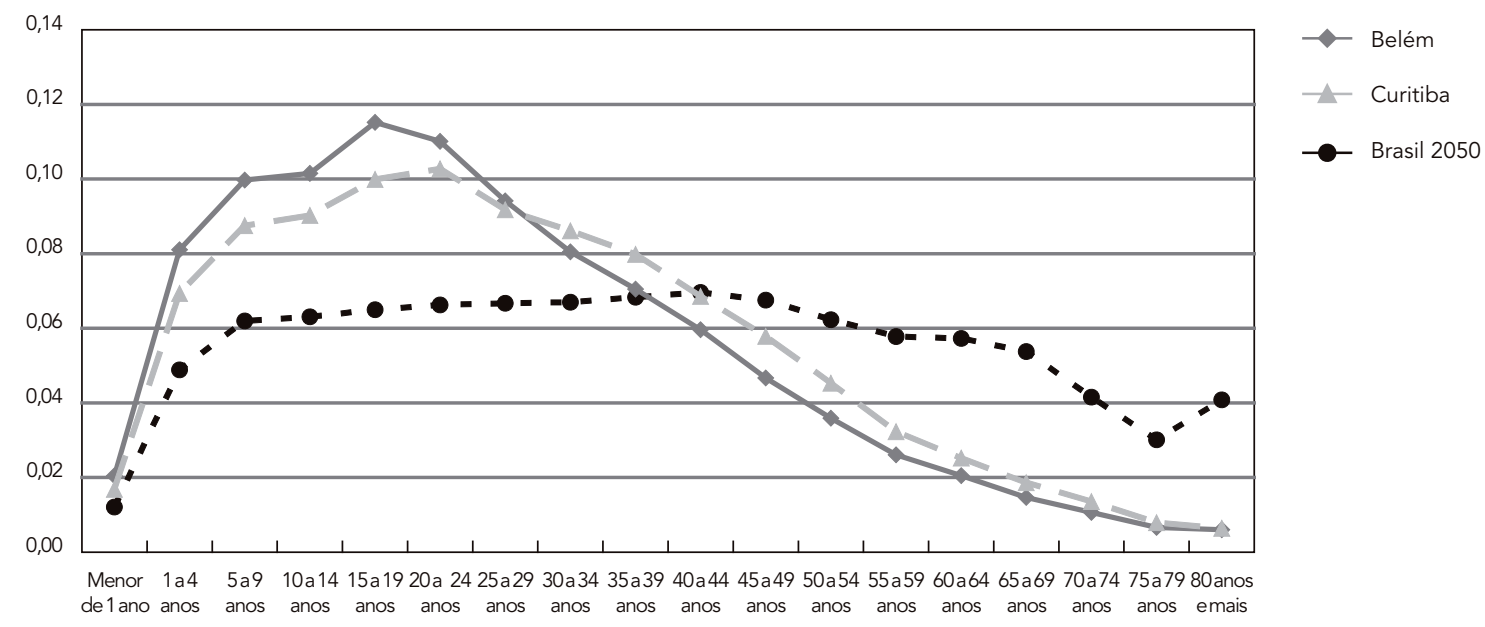

Fonte: Departamento de Informática do SUS 17/Instituto Brasileiro de Geografia e Estatística (Projeções populacionais - revisão 2004. http://www.ibge.gov.br, acessado em 18/Out/2004).

Figura 3

Taxa de utilização de serviço de internação, por grupo etário, entre homens, Belém (Pará) e Curitiba (Paraná), Brasil, 2004.

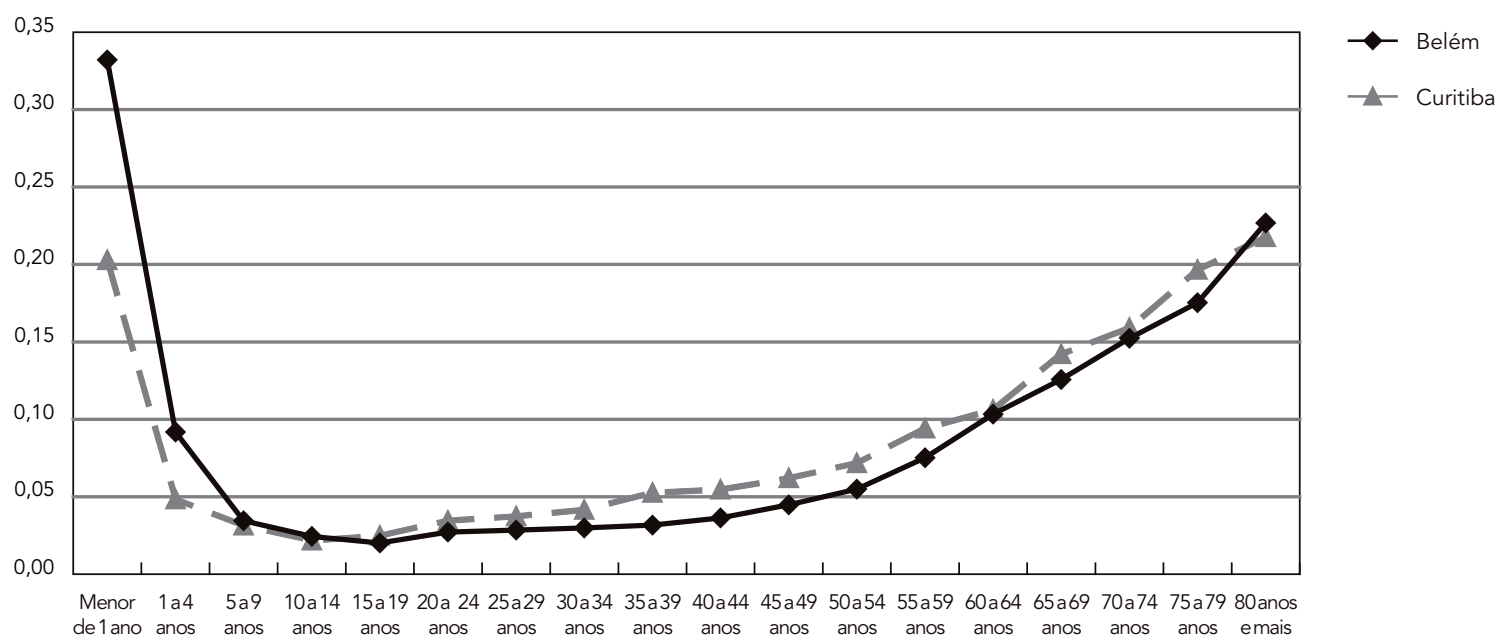

Fonte: Departamento de Informática do SUS 17. 
Figura 4

Porcentagem do gasto total de internação por grupo etário, entre homens, Belém (Pará) e Curitiba (Paraná), Brasil, 2004.

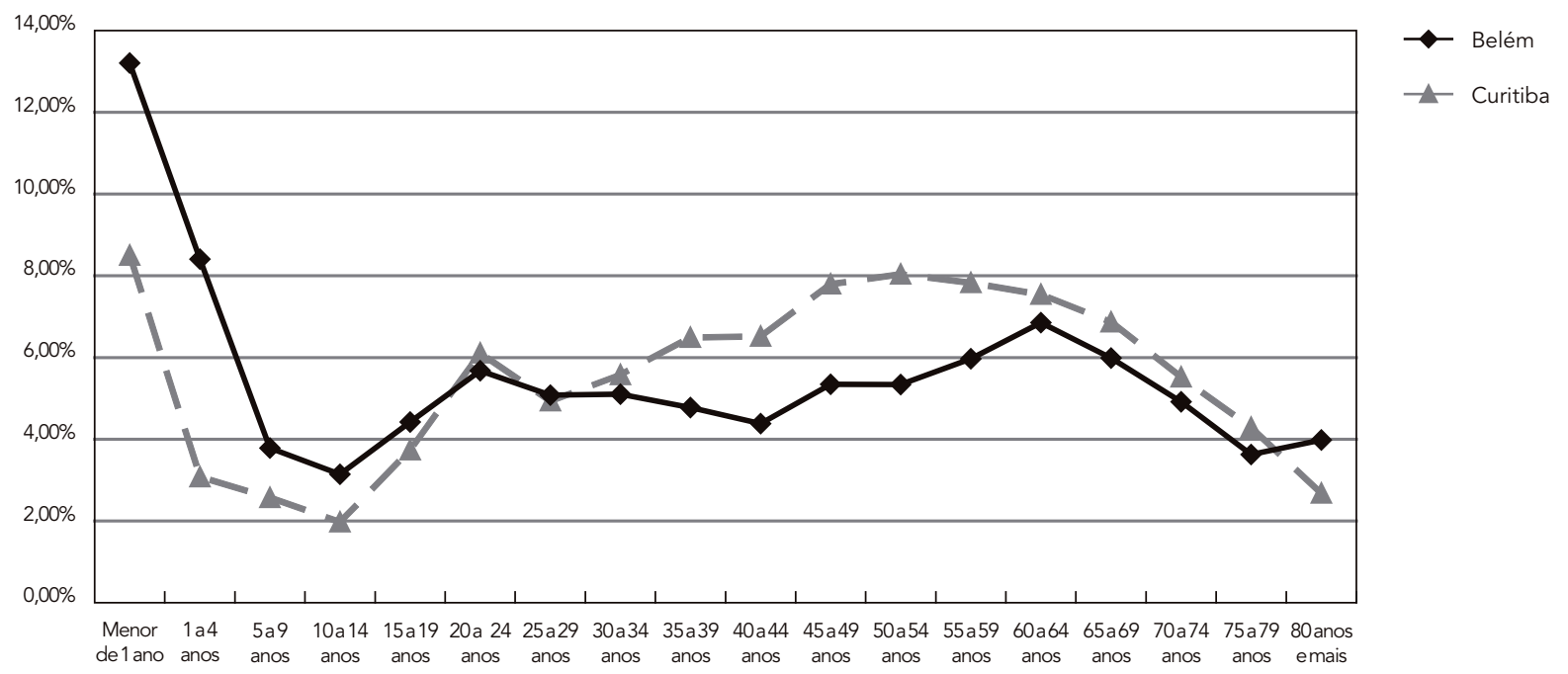

Fonte: Departamento de Informática do SUS 17.

Figura 5

Gasto médio de internação por grupo etário, entre homens, Belém (Pará) e Curitiba (Paraná), Brasil, 2004.

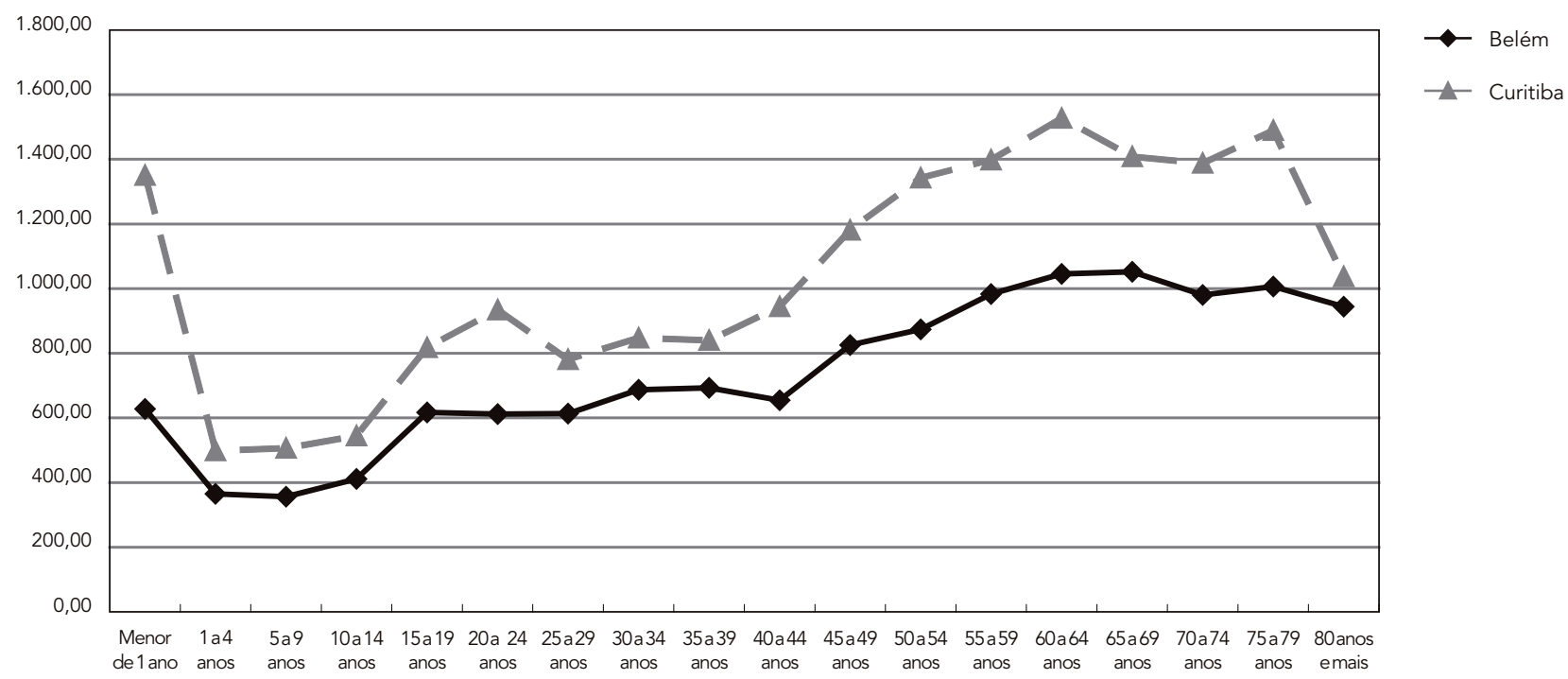

Fonte: Departamento de Informática do SUS 17. 
Resultados da decomposição do gasto total em Belém (Pará) e Curitiba (Paraná) e da simulação com a estrutura etária do Brasil em 2050.

\begin{tabular}{lc}
\hline & $\begin{array}{c}\text { Estrutura etária } \\
\text { observada em } 2004 \\
\text { de Curitiba equivalente } \\
\text { à do Brasil em } 2050\end{array}$ \\
\hline Diferença inicial no gasto total & $17.830 .783,40$ \\
Efeito preço & $12.852 .732,46$ \\
Efeito taxa & $2.713 .208,34$ \\
Efeito composição & $2.264 .842,60$ \\
Proporção da diferença atribuída ao efeito preço (\%) & 72,08 \\
Proporção da diferença atribuída ao efeito taxa (\%) & 15,22 \\
Proporção da diferença atribuída ao efeito composição (\%) & $3.778 .169,89$ \\
\hline
\end{tabular}

Fonte: Departamento de Informática do SUS 17/Instituto Brasileiro de Geografia e Estatística (Projeções populacionais - revisão 2004. http://www.ibge.gov.br, acessado em 18/Out/2004).

de internação tendem a ser mais elevados nas idades mais velhas, fato que não se observa em Belém. No entanto, as curvas para ambas as capitais não apresentam comportamento crescente até o fim do ciclo de vida, com queda dos valores a partir dos 70 anos, confirmando os achados de Nunes 19 para o país como um todo. Este fato pode ser explicado pelos riscos crescentes associados aos procedimentos invasivos aplicados em indivíduos mais velhos, cuja saúde tende a ser mais frágil. Analisando o caso dos homens paulistas, Berenstein 22 verifica que, até a idade de 70 anos, os procedimentos invasivos, como a revascularização cirúrgica do miocárdio com circulação extracorpórea, possuem uma alta freqüência e representam alta parcela do valor total despendido para este grupo etário. À proporção que a idade aumenta, procedimentos invasivos começam a representar uma menor parcela, tanto da freqüência quanto do gasto, dando lugar a procedimentos mais simples, que muitas vezes não requerem cirurgia 24 . Contudo, com o aumento de tecnologia para o tratamento de enfermidades características do grupo etário mais idoso, acredita-se que essa curva deva mudar de perfil, a exemplo do que ocorre em países desenvolvidos, tornando-se crescente até idades cada vez mais avançadas. Como essa é a faixa etária cujo peso relativo na população total é o que mais cresce, a elevação dos gastos médios associados às suas enfermidades é acompanhada da preocupação com o impacto desta tendência nos gastos totais da saúde 3 .

O modelo brasileiro de saúde certamente está muito mais centrado nos serviços curativos do que no atendimento preventivo, uma vez que há muito pouco investimento nas rotinas de pre- venção, e a população, seja por motivos socioculturais ou financeiros, só tende a procurar os serviços de saúde quando já se encontra doente 23 . Se nada for feito nesse sentido, a exemplo dos esforços de outros países que respondem ao processo de envelhecimento com o investimento mais intensivo na prevenção das doenças crônico-degenerativas, os gastos de internação tendem a sofrer forte elevação em conseqüência do aumento da proporção de idosos no país e com o maior desenvolvimento das tecnologias curativas. Outra forma de se evitar um grande número de internações de idosos é oferecer acompanhamento e tratamento ambulatorial adequados. Certas doenças crônicas, como asma, diabetes e hipertensão, podem ser tratadas sem a necessidade de uma internação 24 .

Neste trabalho, constatou-se que Curitiba, que apresenta um grau mais elevado de desenvolvimento, uma melhor oferta de serviços de saúde e uma população mais envelhecida do que Belém, apresenta um perfil de maior concentração dos gastos totais em grupos etários mais avançados. Belém, por outro lado, apresenta uma estrutura de gastos decrescente com a idade. Isso é um indício de que a transição epidemiológica, que se caracteriza pela mudança de um perfil de alta prevalência de doenças infecto-parasitárias para a maior incidência de doenças crônico-degenerativas, faz com que os gastos médios aumentem mais rápido com a idade. Para reverter essa situação é fundamental que se realize um considerável esforço direcionado à prevenção das doenças, especialmente aquelas fortemente associadas a hábitos ao longo de todo ciclo de vida. No caso brasileiro, há ainda o agravante de que se convive, no sistema de saúde, com os casos ainda não 
solucionados de doenças infecciosas e a crescente demanda por tratamento de doenças crônicas, o que gera, conseqüentemente, uma competição por recursos 15 .

Ao decompor o gasto total em efeito preço, efeito taxa e efeito composição, pôde-se verificar que o efeito preço exerce papel determinante nas diferenças de gasto total entre regiões com distintos níveis de desenvolvimento. A diferença nos gastos provém dos níveis tecnológicos oferecidos pelos serviços de saúde à população e também das diferenças de morbidade, uma vez que o processo de transição epidemiológica está mais avançado em Curitiba do que em Belém. Ao potencializar o efeito composição, por meio da estrutura etária do Brasil em 2050, verifica-se que o efeito preço cai, dando lugar a uma maior influência do efeito composição. Contudo, sabese que, com o envelhecimento populacional e o desenvolvimento das regiões, os preços de tratamentos podem aumentar, principalmente nos grupos etários mais idosos, que envolvem um maior tempo de permanência no hospital e tratamentos mais complexos, já que esta população tende a apresentar vários problemas simultane-

\section{Resumo}

O objetivo deste artigo é examinar a importância da composição etária da população sobre os gastos com internação hospitalar no Brasil. São utilizados dados do Sistema de Informação Hospitalar de Curitiba (Paraná) e Belém (Pará), em 2004, para separar o efeito de diferenças na composição etária sobre os gastos com internação no Sistema Único de Saúde, dos efeitos preço (diferenças nos gastos médios por idade) e taxa (diferenças na taxas de utilização por idade). Além disso, o artigo examina o papel do envelhecimento populacional sobre o efeito de composição com base nas mudanças projetadas para a estrutura etária do Brasil em 2050. Os resultados indicam que as diferenças na composição etária explicam apenas $13 \%$ das diferenças atuais dos gastos entre as duas cidades. Aproximadamente $72 \%$ das diferenças nos gastos totais devemse ao efeito preço. No entanto, caso a taxa de utilização e o gasto médio por idade mantenham-se constantes no futuro, o envelhecimento populacional explicará mais da metade da diferença nos gastos totais com internação hospitalar, sugerindo o papel significativo da dinâmica demográfica sobre os gastos futuros com saúde no Brasil.

Gastos em Saúde; Distribuição por Idade; Envelhecimento; Hospitalização amente. Dessa forma, o aumento da proporção de idosos pode gerar uma explosão nos gastos de saúde para a sociedade. Medidas preventivas e tecnologias que visem à redução dos gastos, entretanto, podem conter essa perspectiva.

Este trabalho visa a contribuir para o conhecimento dos componentes do gasto com saúde na rede pública. No entanto, foram analisados somente os gastos com internações, e para se ter um bom planejamento do dispêndio futuro do Sistema Único de Saúde é necessário analisar também os outros tipos de gastos que aqui não foram abordados como, por exemplo, o atendimento ambulatorial. Como o envelhecimento da população está ocorrendo de forma bastante acelerada no Brasil, espera-se que, em um futuro próximo, ocorra um aumento na demanda por serviços de saúde, principalmente de procedimentos ligados à prevenção e tratamento de doenças crônicas. Sendo assim, é necessário compreender o que explica os gastos mais elevados entre os idosos, a fim de que se possa melhorar a saúde deste segmento da população sem a necessidade de um aumento exagerado dos gastos totais.

\section{Colaboradores}

C. K. Berenstein analisou os dados, elaborou a revisão de literatura e redigiu a primeira versão do manuscrito. S. Wajnman participou na idealização do artigo, reformulação, elaboração e na redação da versão final do manuscrito. 


\section{Referências}

1. Zweifel P, Felder S, Meiers M. Ageing of population and health care expenditure: a red herring? Health Econ 1999; 8:485-96.

2. Reinhardt UE. Does the aging of the population really drive the demand for health care? Health Aff (Millwood) 2003; 22:27-39.

3. Wong LLR, Carvalho JAM. O rápido processo de envelhecimento populacional no Brasil: sérios desafios para as políticas públicas. Rev Bras Estud Pop 2006; 23:5-26.

4. Chaimowicz F. Health of the Brazilian elderly population on the eve of the 21st century: current problems, forecasts and alternatives. Rev Saúde Pública 1997; 31:184-200.

5. Roos N, Burchill C, Carriere K. Who are the high hospital users? A Canadian case study. J Health Serv Res Policy 2003; 8:5-10.

6. Cutler DM, Meara E. The medical costs of the young and old: a forty-year perspective. In: Wise DA, editors. Frontiers in the economics of aging. Chicago: University of Chicago; 1998. p. 215-46.

7. Jacobzone S, Oxley H. Ageing and health care costs. Internationale Politik und Gesellschaft [serial online] 2002; (1). http://www.fes.de/ipg/ ipg1_2002/ARTJACOBZONE.htm (acessado em 29/Mar/2004).

8. Pol LG, Thomas RK. The demography of health and health care. 2nd Ed. New York: Kluwer Academic/ Plenum; 2000.

9. Buchner F. The steeping of health expenditure profiles. http://www.actuaries.org/IAAHS/Colloquia/ Dresden/Buchner\%20presentation.pdf (acessado em 20/Mar/2006).

10. Sheiner L. The effects of technology on the age distribution of health spending: a cross-country perspective. https://www.federalreserve.gov/pubs/ feds/2004/200414/200414pap.pdf (acessado em 20/Mar/2006).

11. Miller T. Increasing longevity and Medicare expenditures. Demography 2001; 38:215-26.

12. Spillman BC. Changes in elderly disability rates and the implication for health care utilization cost. Milbank Q 2004; 82:157-94.

13. Seshamani M, Gray A. The impact of ageing on expenditures in the National Health Service. Age Ageing 2002; 31:287-94.
14. Berquó E, Cavenaghi S. Mapeamento socioeconômico e demográfico dos regimes de fecundidade no Brasil e sua variação entre 1991 e 2000. In: Anais do Encontro Nacional de Estudos Populacionais. Caxambu: Associação Brasileira de Estudos Populacionais; 2004. p. 14.

15. Saad P. O envelhecimento populacional e seus reflexos na área da saúde. In: Anais do VII Encontro de Estudos Populacionais. v. 3. Caxambu: Associação Brasileira de Estudos Populacionais; 1990. p. 353-70.

16. Veras RP. Brazil is getting older: demographic changes and epidemiological challenges. Rev Saúde Pública 1991; 25:476-88.

17. Departamento de Informática do SUS. Sistema de informação hospitalar (SIH). Autorização de Internação Hospitalar - AIH [CD-ROM]. Brasília: Ministério da Saúde; 2004.

18. Departamento de Informática do SUS. Pesquisa de assistência médico-sanitária (AMS) [CD-ROM]. Brasília: Ministério da Saúde; 1999.

19. Nunes A. O envelhecimento populacional e as despesas do Sistema Único de Saúde. In: Camarano AA, organizador. Os novos idosos brasileiros. Muito além dos 60? Rio de Janeiro: Instituto de Pesquisa Econômica Aplicada; 2004. p. 427-50.

20. Preston SH, Heuveline P, Guillot M. Demography: measuring and modeling population processes. Malden: Blackwell; 2001.

21. Hulka BS, Wheat JR. Patterns of utilization - the patient perspective. Med Care 1985; 23:438-60.

22. Berenstein CK. O perfil etário dos custos de internação na saúde pública no Brasil: uma análise para as capitais das regiões metropolitanas do Brasil em 2000 [Dissertação de Mestrado]. Belo Horizonte: Universidade Federal de Minas Gerais; 2005.

23. Heimann LS, Ibanhes LC, Barboza R, organizadores. O público e o privado na saúde. São Paulo: Editora Hucitec; 2005.

24. Bindman AB, Grumbach K, Osmond D, Komaromy M, Vranizan K, Lurie N, et al. Preventable Hospitalizations and Access to Health Care. JAMA 1995; 274:305-11.

Recebido em 01/Jun/2006

Versão final reapresentada em 03/Mar/2008

Aprovado em 18/Mar/2008 